\title{
A Fixed Point Theorem for Left Amenable Semi-Topological Semi Groups
}

\section{Naderi F*}

Department of Mathematics, Tarbiat Modares University, Iran

\begin{abstract}
In this note, we extend and improve the corresponding result of Takahashi. Explanation of DeMarr's theorem is further generalized for some semi groups of non-expansive self- maps on $\mathrm{K}$ by the following considerations which are explained in the paper. The application of Zorn's lemma and its application are explained. An application of Zorn's lemma shows that there exists a minimal non-empty compact convex and S-invariant subset.
\end{abstract}

Keywords: Non-expansive mappings; Semi-topological semi groups; Amenable; Left reversible

\section{Introduction}

Let $\mathrm{K}$ be a subset of a Banach space $\mathrm{E}$. A self-mapping $\mathrm{T}$ on $\mathrm{K}$ is said to be non- expansive if $\|\mathrm{T}(\mathrm{x})-\mathrm{T}(\mathrm{y})\| \leq\|\mathrm{x}-\mathrm{y}\|$ for all $\mathrm{x}, \mathrm{y} \in \mathrm{K}$. In [1] DeMarr proved the following theorem:

Theorem 1.1: For any non-empty compact convex subset $\mathrm{K}$ of a Banach space E, each commuting family of non-expansive selfmappings on $\mathrm{K}$ has a common fixed point in $\mathrm{K}$.

DeMarr's theorem can be further generalized for some semigroups of non-expansive self- maps on $\mathrm{K}$ by the following considerations.

Let $\mathrm{S}$ be a semi-topological semigroup, i.e. $\mathrm{S}$ is a semigroup with a Hausdorff topology such that for each a $\in \mathrm{S}$, the mappings $\mathrm{s} 1 \mapsto s a$ and $s \mapsto a s$ from $S$ into $S$ are continuous. $S$ is called left reversible if any two closed right ideals of $S$ have non-void intersection. Let $l^{\infty}(S)$ be the $C^{*}$-algebra of all bounded complex-valued functions on $S$ with supremum norm and point-wise multiplication. For each $s \in S$ and $f \in l^{\infty}(S)$, denote by $l_{s}(f)$ and $r_{s}(f)$ the left and right translates of $f$ by $s$ respectively, that is $\operatorname{li}_{\mathrm{s}} \mathrm{f}(\mathrm{t})=\mathrm{f}(\mathrm{st})$ and $\mathrm{r}_{\mathrm{s}} \mathrm{f}(\mathrm{t})=\mathrm{f}(\mathrm{ts})$ for all $\mathrm{t} \in \mathrm{S}$. Let $\mathrm{X}$ be a closed subspace of $l^{\circ}(S)$ containing constants and be invariant under translations. Then a linear functional $\mathrm{m} \in \mathrm{X}^{*}$ is called a mean if $\|\mathrm{m}\|=\mathrm{m}(1)=1$, and a left invariant mean (LIM) if moreover $m\left(l_{s}(f)\right)=m(f)$ for $s \in S, f \in X$. Let $C_{b}(S)$ be the space of all bounded continuous complex-valued functions on $S$ with supremum norm and LUC (S) be the space of left uniformly continuous functions on S, i.e., all functions $f \in C_{b}(S)$ for which the mapping $s \rightarrow l_{s} f: S \rightarrow C_{b}(S)$ is continuous when $C_{b}(S)$ has the sup-norm topology. Then LUC(S) is a $\mathrm{C} *$-subalgebra of $\mathrm{C}_{\mathrm{b}}(\mathrm{S})$ invariant under translations and containing constant functions. $\mathrm{S}$ is called left amenable if LUC (S) has a LIM. The space of all right uniformly continuous functions, RUC(S), and right amenability are defined similarly. The semi-topological semigroup $\mathrm{S}$ is called amenable if it is both left and right amenable, in this situation there is a mean which is both left and right invariant. Left amenable semi-topological semigroups include commutative semigroups, as well as compact and solvable groups. The free (semi)group on two or more generators is not left amenable. When $S$ is discrete, $\operatorname{LUC}(S)=l^{\infty}(S)$ and (left) amenability of $S$ yields the (left) reversibility of S. For more details on amenability, examples and relations [2-5].

An action of $\mathrm{S}$ on a topological space $\mathrm{E}$ is a mapping $(s, x) \mapsto s(x)$ from $S \times E$ into $E$ such that $(s t)(x)=s(t(x))$ for $s, t \in S, x \in E$. The action is separately continuous if it is continuous in each variable when the other is kept fixed. Every action of $\mathrm{S}$ on $\mathrm{E}$ induces a representation of $S$ as a semigroup of self-mappings on $E$ denoted by $S$, and the two semigroups are usually identified. When the action is separately continuous, each member of $\mathrm{S}$ is a continuous mapping on $\mathrm{E}$. A subset $\mathrm{K} \subseteq \mathrm{E}$ is called $\mathrm{S}$-invariant if $\mathrm{sK} \subseteq \mathrm{K}$ for each $\mathrm{s} \in \mathrm{S}$. We say that $\mathrm{S}$ has a common fixed point in $\mathrm{E}$, if there exists a singleton S-invariant subset of $\mathrm{E}$. When $\mathrm{E}$ is a normed space the action of $\mathrm{S}$ on $\mathrm{E}$ is called nonexpansive if $\|s(x)-s(y)\| \leq\|x-y\|$ for all $s \in S$ and $x, y \in E$.

Takahashi [6] proved a generalization of DeMarr's fixed point theorem as follows:

Theorem 1.2: Let $\mathrm{K}$ be a non-empty compact convex subset of a Banach space $\mathrm{E}$ and $\mathrm{S}$ be an amenable discrete semigroup which acts on $\mathrm{K}$ separately continuous and non-expansive. Then $\mathrm{S}$ has a common fixed point in K. It is well-known that every left amenable discrete semigroup is left reversible [4], so Mitchell [7] proved the following theorem:

Theorem 1.3: Let $\mathrm{K}$ be a non-empty compact convex subset of a Banach space $\mathrm{E}$ and $\mathrm{S}$ be a left reversible discrete semigroup which acts on $\mathrm{K}$ separately continuous and non- expansive. Then $\mathrm{S}$ has a common fixed point in K. But it is not the case that all left amenable semitopological semigroups are left reversible as the following example shows [4]:

Example 1.4: Let $S$ be a topological space which is regular and Hausdorff. Then $\mathrm{C}_{b}(\mathrm{~S})$ consists of constant functions only. Define on $S$ the multiplication $s t=s$ for all $s, t \in S$. Let $a \in S$ be fixed. Define $\mu(f)=f$ (a) for all a $\in S$. Then $\mu$ is a left invariant mean on $C(S)$, but $S$ is not reversible.

Now the question naturally arises as to whether this is true if one considers a left amenable semi-topological semigroup in Takahashi's theorem.

In this paper, we show that the answer is affirmative. Our theorem is new and is not a result of any previous work.

\section{Main Theorem}

The space of almost periodic functions is the space of all $f \in C(S)$ such that $\left\{l_{\mathrm{s}} \mathrm{f}: \mathrm{s} \in \mathrm{S}\right\}$ is relatively compact in the sup-norm topology of $\mathrm{C}(\mathrm{S})$ and is denoted by $\mathrm{AP}(\mathrm{S})$. For any semi-topological semigroup $\mathrm{S}$ we have the following theorem [1].

Theorem 2.1: (a)f $\in \operatorname{AP}(S)$ if and only if $\left\{\mathrm{r}_{\mathrm{s}} \mathrm{s}, \mathrm{s} \in \mathrm{S}\right\}$ is relatively compact in the sup-norm topology of $\mathrm{C}(\mathrm{S})$.

*Corresponding author: Naderi F, Department of Mathematics, Tarbiat Modares University, Iran, Tel: 00989127309428; E-mail: f.naderi@modares.ac.ir

Received April 12, 2015; Accepted May 14, 2015; Published May 22, 2015

Citation: Naderi F (2015) A Fixed Point Theorem for Left Amenable SemiTopological Semi Groups. J Phys Math 6: 138. doi:10.4172/2090-0902.1000138

Copyright: (c) 2015 Naderi F. This is an open-access article distributed under the terms of the Creative Commons Attribution License, which permits unrestricted use, distribution, and reproduction in any medium, provided the original author and source are credited. 
(b) $\mathrm{AP}(\mathrm{S}) \subseteq \mathrm{LUC}(\mathrm{S}) \cap \mathrm{RUC}(\mathrm{S})$.

The following lemma is important in proving our main theorem and lets one replace the discrete semigroup in Takahashi's theorem by a general semi-topological semigroup.

Lemma 2.2: Let $S$ be a semi-topological semigroup which acts separately continuous and non-expansive on a compact subset $\mathrm{M}$ of a Banach space $E$. Then for each $\mathrm{m} \in \mathrm{M}$ and each $\mathrm{f} \in \mathrm{C}(\mathrm{M})$ we have $\mathrm{f}_{\mathrm{m}} \in$ LU C (S) where $\mathrm{f}_{\mathrm{m}}(\mathrm{s})=\mathrm{f}(\mathrm{sm})(\mathrm{s} \in \mathrm{S})$.

Proof: For $\mathrm{f} \in \mathrm{C}(\mathrm{M})$ define a new function $\mathrm{A}: \mathrm{M} \rightarrow \mathrm{C}(\mathrm{S})$ by $A(m)=f_{m}$ so $A(m)(s)=f(s m)$ for all $s \in S$. Put sup-norm topology on $C(S)$. We show that $A$ is continuous. Given $m \in M, \varepsilon>0$ we must find a suitable neighborhood for $\mathrm{m}$ such that for all $\mathrm{m}^{\prime}$ in it the inequality $\left\|A\left(m^{\prime}\right)-A(m)\right\|<\varepsilon$ holds. By continuity of $\mathrm{f}$ and compactness of $\mathrm{M}$ the function $\mathrm{f}$ is uniformly continuous, so there is a positive number $\delta$ such that if $\mathrm{u}, \mathrm{v} \in \mathrm{M}$ and $\|\mathrm{u}-\mathrm{v}\|<\delta$, then $|f(u)-f(v)|<\frac{\varepsilon}{2}$. By Archimedean property of numbers, there is a natural number $\mathrm{k}$ for which $\frac{1}{k}<\delta$. For each $m^{\prime}$ in the ball $B\left(m, \frac{1}{k}\right)$ and each $s \in \mathrm{S}$ we have

$$
\left\|s m^{\prime}-s m\right\| \leq\left\|m^{\prime}-m\right\|<\delta<\frac{1}{k}
$$

because the action is non-expansive. Now use uniform continuous property of $\mathrm{f}$ to get $\left|f\left(\mathrm{sm}^{\prime}\right)-f(\mathrm{sm})\right|<\varepsilon$. Hence corresponds to $\varepsilon>0$ we found the ball $B\left(m, \frac{1}{k}\right)$ so that if $m^{\prime} \in B(m, 1)$, then

$$
\left|f\left(s m^{\prime}\right)-f(s m)\right|=\left|A\left(m^{\prime}\right)(s)-A(m)(s)\right|<\frac{\varepsilon}{2}
$$

for all $s \in S$. Consequently

$$
\left\|A\left(m^{\prime}\right)-A(m)\right\|=\sup \left\{\left|A\left(m^{\prime}\right)(s)-A(m)(s)\right|: s \in S\right\}<\frac{\varepsilon}{2}
$$

which shows that $\mathrm{A}$ is continuous. On the other hand for each right translate of $\mathrm{f}_{\mathrm{m}}=\mathrm{A}(\mathrm{m})$ we have

$$
\mathrm{r}_{\mathrm{a}}\left(\mathrm{f}_{\mathrm{m}}\right)(\mathrm{s})=\mathrm{f}_{\mathrm{m}}(\mathrm{sa})=\mathrm{f}(\mathrm{sam})=\mathrm{f}_{\mathrm{am}}(\mathrm{s})=\mathrm{A}(\mathrm{am})(\mathrm{s}) ; \mathrm{s}, \mathrm{a} \in \mathrm{S}
$$

that is $\mathrm{r}_{\mathrm{a}} \mathrm{A}(\mathrm{m})=\mathrm{A}(\mathrm{am})$ hence $\left\{\mathrm{r}_{\mathrm{a}} \mathrm{f}_{\mathrm{m}}: \mathrm{a} \in \mathrm{S}\right\}=\mathrm{A}(\mathrm{Sm})$. The set $\mathrm{Sm}$ is relatively compact in $\mathrm{M}$ and $\mathrm{A}$ is continuous, so $\mathrm{A}(\mathrm{Sm})$ is relatively compact in the sup-norm topology of $\mathrm{C}(\mathrm{S})$. Therefore by theorem 2.1 part (a) we see that $\mathrm{f}_{\mathrm{m}}=A(\mathrm{~m}) \in A P(S)$ and from part (b) $\mathrm{f}_{\mathrm{m}} \in \mathrm{LU} C(\mathrm{~S})$.

Now we use the above lemma to modify Takahashi's proof [7] for left amenable semi- topological semigroups which are not necessarily discrete.

Theorem 2.3: Let $\mathrm{K}$ be a non-empty compact convex subset of a Banach space $\mathrm{E}$ and $\mathrm{S}$ be a left amenable semi-topological semigroup which acts on $\mathrm{K}$ separately continuous and non-expansive. Then $\mathrm{S}$ has a common fixed point in $\mathrm{K}$.

Proof: An application of Zorn's lemma shows that there exists a minimal non-empty compact convex and S-invariant subset $\mathrm{X} \subseteq \mathrm{K}$. If $\mathrm{X}$ is a singleton we are done, otherwise apply Zorn's lemma for the second time to get a minimal non-empty compact and S- invariant subset $\mathrm{M} \subseteq \mathrm{X}$.

We claim that $M$ is $S$-preserved, i.e. $M=s M$ for all $s \in S$. Let $v$ be a left invariant mean on LUC (S) and define $\mu(f)=v\left(f_{m}\right)$, where $f_{m}$ is defined as in lemma 2.2. Then by Riesz representation theorem, $\mu$ induces a regular probability measure on $M$ (still denoted by $\mu$ ) such that $\mu(\mathrm{sB})=\mu(\mathrm{B})$ for all Borel sets $\mathrm{B} \subseteq \mathrm{M}$ and $\mathrm{s} \in \mathrm{S}$. Let $\mathrm{F}$ be the support of $\mu$. Each $s \in S$ defines a measurable continuous function from $M$ into $M$, so by basic properties of support $F \subseteq s M, \mu(s M)=\mu(M)=1$ [7]. Assume that $\chi_{\mathrm{F}}$ is the characteristic function of $F$. For each $s \in S$,

$$
1=\mu(F)=\int_{M} \chi F(y) d \mu=\int_{M} \chi_{F}(s y) d \mu=\mu\left(s^{-1} F\right)
$$

$\left(\mathrm{s}^{-1} \mathrm{~F}\right.$ means the pre-image of $\mathrm{F}$ under $\left.\mathrm{s}\right)$ again by the definition and properties of support we see that $\mathrm{F} \subseteq \mathrm{s}^{-1} \mathrm{~F}$, meaning that $\mathrm{F}$ is $\mathrm{S}$-invariant. Hence $\mathrm{F}=\mathrm{M}$ by the minimality of $\mathrm{M}$. Consequently $\mathrm{M}=\mathrm{F} \subseteq \subseteq \mathrm{sM}$ for each $\mathrm{s} \in \mathrm{S}$. But $\mathrm{M}$ was already $\mathrm{S}$-invariant, so $\mathrm{sM}=\mathrm{M}$ for each $\mathrm{s}$ in $\mathrm{S}$.

Now if $M$ is singleton we are done, otherwise if $\delta(M)=\operatorname{diam}(M)$ $>0$, we get a contradiction by DeMarr's lemma [1] which implies that

$$
\exists u \in \overline{c o}(M) \text { such that } \mathrm{r}_{0}=\sup \{\|\mathrm{m}-\mathrm{u}\|: \mathrm{m} \in \mathrm{M}\}<\delta(\mathrm{M}) .
$$

Define $X_{0}=\bigcap_{m \in M} B\left[m, r_{0}\right]$, then $\mathrm{X}_{0}$ is a non-empty (indeed $\mathrm{u} \in$ $\mathrm{X} 0$ ) compact convex proper subset of $\mathrm{X}$ such that $\mathrm{s} \mathrm{X}_{0} \subseteq \mathrm{X}_{0}$ for each $\mathrm{s}$ in $\mathrm{S}$ (the inclusion follows from the fact that $\mathrm{M}$ is S-preserved). But this contradicts the minimality of $\mathrm{X}$. Therefore $\mathrm{M}$ contains only one point which is a common fixed point for the action of $S$.

Obviously every amenable discrete semigroup is a left amenable semi-topological semi- group, so we can deduce Takahashi's theorem from our theorem:

Corollary 2.4: Let $\mathrm{K}$ is a non-empty compact convex subset of a Banach space $\mathrm{E}$ and $\mathrm{S}$ is an amenable discrete semigroup which acts on $\mathrm{K}$ separately continuous and non-expansive. Then $\mathrm{S}$ has a common fixed point in $\mathrm{K}[6]$.

\section{References}

1. DeMarr R (1963) Common fixed points for commuting contraction mappings. Pacific J Math 13: 1139-1141.

2. Berglund JF, Junghen HD, Milnes $P$ (1989) Analysis on semi groups. John Wiley \& Sons Inc., New York, USA.

3. Day MM (1957) Amenable semi groups. Illinois J Math 1: 509-544.

4. Lau ATM (2010) Normal structure and common fixed point properties for sem groups of non-expansive Mappings in Banach spaces. Fixed Point Theory and Application.

5. Paterson AL (1988) Amenability. American Mathematical Society, Providence

6. Takahashi W (1969) Fixed point theorem for amenable semi group of nonexpansive mappings. Kodai Math Sem Rep 21: 383-386.

7. Mitchell T (1970) Fixed points of reversible semi groups of non-expansive mappings. Kodai Math Sem Rep 22: 322-323.

Citation: Naderi F (2015) A Fixed Point Theorem for Left Amenable SemiTopological Semi Groups. J Phys Math 6: 138. doi:10.4172/2090-0902.1000138 